Research article

\title{
Larkin: FDA Emergency Use Authorized Treatment Protocol of DeltaRex-G for Severe Covid-19 [IND\# 27185]
}

Ellie Soheili ${ }^{1}$, George Michel' ${ }^{1}$, B. Almila Yerli², Inderpreet Bhatti ${ }^{3}$, Ted T. Kim², Don A. Brigham ${ }^{4}$, Sant P. Chawla ${ }^{2}$, Frederick L. Hall ${ }^{5}$, Erlinda M. Gordon ${ }^{2,4,5}$

1. Larkin Community Hospital, South Miami, FL, USA

2. Cancer Center of Southern California, Santa Monica CA, USA

3. Scripps Clinic, San Diego, CA

4. Aveni Foundation, Santa Monica CA

5. Delta Next-Gene, LLC, Santa Monica CA, USA

Corresponding Author: Dr. Erlinda M. Gordon, Cancer Center of Southern California, 2811 Wilshire Boulevard, Suite 414, Santa Monica, CA 90403, USA

Received: October 08, 2021; Accepted: October 26, 2021; Published: October 29, 2021

\begin{abstract}
Background: Histopathologic examination of lung biopsies from patients with COVID-19 complicated by acute respiratory distress syndrome (ARDS) and lung cancer has shown striking similarities providing the rationale for using DeltaRex-G in COVID-19 induced ARDS. DeltaRex-G is a disease-targeted replication-incompetent amphotropic MLV-based retrovector. Mechanistically, DeltaRex G displays a collagen (XC)-binding peptide on its surface for targeting abnormal $\mathrm{XC}$ proteins exposed in the damaged lung environment (DLE), as well as in the tumor microenvironment (TME) of lung cancer. Injected intravenously, DeltaRex-G nanoparticles seek out and accumulate in the DLE or TME where abnormal $\mathrm{XC}$ proteins are exposed, in the vicinity of activated/proliferative cells. Hypothesis: DeltaRex-G enters the cells via their native viral receptors, integrate into the genome of rapidly dividing $\mathrm{T}$ cells and activated macrophages, and kill them by blocking the G1 phase of the cell division cycle, and in turn, reduce the cytokine storm driving overall severity of ARDS. Objectives: (1) To improve survival from severe COVID-19, (2) To hasten recovery from severe COVID-19. Treatment Plan: The patient will receive DeltaRex-G intravenously at a dose of $1.2 \times 10 \mathrm{e} 11$ colony forming units (CFU) daily for 7 days. DeltaRex-G is currently available as Expanded Access for an intermediate size population with advanced pancreatic cancer and sarcoma as well as individual patients with breast, prostate, lung, bile duct and skin cancer (IND\# 19130), and has gained Emergency Use Authorization for a patient with severe COVID-19 induced ARDS (IND\# 27185).
\end{abstract}

Keywords: FDA emergency use authorization, COVID-19, acute respiratory distress syndrome, DeltaRex-G, gene therapy, immunomodulation, cytokine release syndrome, cytokine storm

\section{Introduction}

According to the Center for Disease Control and Prevention, more than 38 million COVID-19 cases have been reported in the United States as of August 31, 2021 [1]. There have been over 2 million $(2,674,920)$ COVID hospitalizations and 631,440 deaths reported [1]. COVID-19 associated ARDS has been shown to be the primary mortality cause among patients hospitalized in the setting of severe COVID-19 infection [2]. We propose DeltaRex-G, a disease-targeted replication-incompetent retroviral vector that ultimately works by accumulating in inflamed lung tissue and inhibiting the hyperproliferation of immune cells, as a potential upfront treatment modality in COVID-19 complicated by ARDS. This article is dedicated to the treatment of symp- tomatic COVID-19 disease, explaining the rationale behind DeltaRex-G treatment in COVID-19 induced ARDS, and providing the treatment protocol which has gained US FDA Emergency Use Authorization for an adult patient with severe COVID-19 induced ARDS (IND\# 27185). This EUA, granted within 24 hours of application with the FDA, provides a precedent for the treatment of other patients afflicted with severe COVID-19.

\section{Background and Rationale}

Acute respiratory distress syndrome (ARDS) is highly associated with mortality and morbidity in patients with COVID-19. In a global literature survey, $33 \%$ of hospitalized patients diag- 
nosed with severe COVID-19 were found to develop ARDS, and the mortality rate in COVID-19 associated ARDS was found to be as high as $45 \%$. The incidence of ARDS found in patients who died due to COVID-19 was as high as 90\% [3], further indicating a significant need for advancement in the current standardized treatment approach.

Since first described in 1967, there remains a lack of standardized pharmacological treatment for ARDS proven to be beneficial, and the mortality rate due to ARDS due to any cause is still as high as $40 \%$ [4]. The reason behind disease progression from a mild disease to ARDS in patients with COVID-19 is thought to be an overstimulation of immune cells and the profound release of pro-inflammatory cytokines, which results in further immune cell infiltration and tissue destruction [5]. Cytokine release syndrome or cytokine storm is defined as severe systemic inflammation, hemodynamic instability, multiorgan failure, with high chance of potential death [6], initially presenting locally in the lungs due to activated lung macrophages. This results in a hyperimmune response and ultimately leads to a severe cytokine leak into the systemic circulation, progressing a controlled localized response to an uncontrollable systemic process. With a lack of containment, patients are seen to have rapid clinical deterioration resulting in a significant increase in overall mortality depending on the severity of disease and precipitated by factors that predispose patients to an increased susceptibility such as other medical co-morbidities including but not limited to, obesity, hypertension, and other immunocompromising illnesses [7]

Post-mortem studies of patients with severe COVID-19 showed significant endothelial cell damage, thrombosis, microangiopathy, and angiogenesis in the lung tissue [8]. Histopathology of ARDS of lungs demonstrated bilateral diffuse alveolar damage, hyaline membranes, and inflammation, immune cell infiltration and activation (especially lymphocytes and macrophages) [9]. Widespread damage to the lungs is not thought to be caused by the cytotoxic effect of SARS-COV-2 alone. Rather, proinflammatory cytokine production and complement pathway activation further induces endothelial cell damage, which results in thrombosis and inflammation [8,9].

Interestingly, the histopathology of the tumor microenvironment of lung cancer has been found to have striking similarities with that seen in the damaged lung environment (DLE) of COVID-19 associated ARDS. Interaction between lung tumor and extracellular matrix creates inflammation and tissue destruction by increasing oxidative stress and expression of pro-inflammatory cytokines, activating metalloproteinases, and causing matrix degradation resulting in alveolar destruction and fibrosis. For example, newly secreted ECM proteins like type I collagen are abundant in tissues with fibrosis. Therefore, the tumor microenvironment shows similarity to the stroma of wound healing, consisting of significant inflammation and remodeling [10].

DeltaRex-G, an XC-targeted MLV-based retroviral gene delivery system, displays a decapeptide on its surface that hunts down and binds to exposed collagen in injured tissues, accumulates only in diseased areas, in the vicinity of proliferative cancer or immune cells, thus augmenting effective drug concentration with no collateral damage to normal organs. The integration into the genome of a cytocidal human cyclin G1 inhibitor gene into rapidly dividing immune cells inhibits cell proliferation by blocking the G0-G1 phase of the cell division cycle $[11,12]$. In preclinical proof of concept studies, a rat carotid injury model of vascular restenosis and a nude mouse model of liver metastasis have shown that DeltaRex-G (former name: Rexin-G, Mx-dnG1, dnG1) accumulates at sites where exposed collagen is abundant [13]. Apoptosis of proliferative cancer cells and neovasculature by DeltaRex-G was later confirmed in the clinical setting by four Phase 1 and 2 trials for sarcoma, pancreatic cancer, and breast cancer, and in one Phase 2 study for osteosarcoma in the United States [14]. The off the shelf DeltaRex-G vector is not immunogenic and therefore allows repeated infusions without compromise of patient safety. Moreover, the vector allows transduction only of proliferative cells leaving normal differentiated cells intact, resulting in minimal, if any, systemic toxicity [15].

\section{Hypothesis}

DeltaRex-G enters the cells via their native viral receptors, integrates into the genome of rapidly dividing $\mathrm{T}$ cells and activated macrophages, and kills them by blocking the G0-G1 phase of the immune cell division cycle, consequently reducing cytokine release, hastening clinical recovery and improving survival of patients from COVID-19 induced ARDS.

\section{Objectives}

\section{Primary Objective}

To improve survival from severe COVID-19

\section{Secondary objectives}

To hasten recovery from severe COVID-19

\section{Treatment plan}

Rationale for the proposed dose:

a. This is the lowest dose level used in US-based cancer clinical trials $[11,14,16,17]$ with no serious adverse events, i.e., safe in patients with metastatic disease.

b. Using the empirical calculus of parity formula: Assuming the number lymphocytes in $70 \mathrm{~kg}$ man to be $1000 / \mathrm{ul}$ or $1 \mathrm{x} 10 \mathrm{e} 9 /$ liter or $5 \times 10 \mathrm{e} 9 / 5$ liters, and an MOI of 100 (since the vector is diluted in the circulatory system); the amount of vector needed to kill the activated lymphocytes in the damaged lung/organs would be $500 \times 10 \mathrm{e} 9$ or $5 \times 10 \mathrm{e} 11 \mathrm{cfu}$ (estimated required total dose)

c. DRG-101 vector neo titer $=2 \times 10 \mathrm{e} 9 \mathrm{cfu} / \mathrm{ml}$ or $120 \mathrm{cfu} \times$ $10 \mathrm{e} 9 / 60 \mathrm{ml}$ or $1.2 \times 10 \mathrm{e} 11 / 60 \mathrm{ml}$

d. If $60 \mathrm{ml}$ DRG-101 were given i.v. QD x 7 days $=8.4 \times 10 \mathrm{e} 11$ cfu (total dose of DRG-101) will meet the calculus of parity.

e. The 7-day daily dosing was based on the remdesivir trial for severe COVID-19 wherein remdesivir was given for 5 or 10 days [18], and the safety of DeltaRex-G when given daily for 10 days in patients with chemotherapy resistant advanced cancer $[12,19$

\section{Dose and schedule}

The patient will receive DeltaRex-G intravenously (IV) at a dose of 1.2 x 10e11 colony forming units (cfu) $(60 \mathrm{ml})$ per dose daily for 7 days. If the patient develops a treatment-related $>$ Grade 3 adverse event (CTCAE Vs 5.0), the DeltaRex-G infusions will be held and the patient will be monitored until the toxicity has resolved to $<$ Grade 1 , and the patient is stable, after 
which treatment may be resumed. If the adverse event does not resolve to $<$ Grade 1 within 3 days, the DeltaRex-G treatment will be held until the data is discussed with the Food and Drug Administration (FDA) and a decision is made whether to continue or terminate the treatment.

\section{Route of administration}

Preparation and Infusion of DeltaRex-G retrovector: The vector is stored at minus 65 to minus $90^{\circ} \mathrm{C}$ freezer until used. Fifteen minutes before infusion, the product is thawed in a $37^{\circ} \mathrm{C}$ water bath and immediately infused upon thawing. Patient will receive infusions of DeltaRex-G via a peripheral vein or a central IV line by slow IV infusion at $4 \mathrm{~mL}$ per minute.

\section{Other concomitant medications}

Thirty minutes prior to vector infusion: Acute reaction prophylactic therapy consists of dexamethasone, $2 \mathrm{mg}$ IV over 30 minutes or p.o. during or post-infusion, diphenhydramine $25-50$ mg intravenously or orally may be given for acute hypersensitivity reactions and meperidine $25-50 \mathrm{mg}$ intravenously may be given for chills. Non- steroidal anti-inflammatory drugs, such as ibuprofen $400 \mathrm{mg}$ every 6 hours orally may be used as needed for pain and/or fever unless contraindicated.

\section{Evaluations and monitoring}

Primary and secondary outcome measures used in "Study to Evaluate the Safety and Antiviral Activity of Remdesivir (GS5734TM) in Participants With Severe Coronavirus Disease (COVID-19)" NCT04292899, will be used [18].

\section{Primary outcome measures}

a. The Odds of Ratio for Improvement on a 7-point Ordinal Scale on Day 14 [ Time Frame: Day 14 ]

The odds ratio represents the odds of improvement in the ordinal scale. The ordinal scale is an assessment of the clinical status on a given day. Each day, the worst score from the previous day will be recorded.

The scale is as follows:

1. Death

2. Hospitalized, on invasive mechanical ventilation or Extracorporeal Membrane Oxygenation (ECMO);

3. Hospitalized, on non-invasive ventilation or high flow oxygen devices;

4. Hospitalized, requiring low flow supplemental oxygen;

5. Hospitalized, not requiring supplemental oxygen - requiring ongoing medical care (coronavirus (COVID-19) related or otherwise);

6. Hospitalized, not requiring supplemental oxygen - no longer required ongoing medical care;

7. Not hospitalized.

\section{Secondary outcome measures}

Percentage of Participants Who Experienced Treatment-Emergent Adverse Events (TEAEs) [Time Frame: First dose date up to last dose date (maximum: 10 days) plus 30 days]

Treatment-emergent Adverse Events (TEAE) were defined as AEs with onset dates on or after the study treatment start date and no later than 30 days after the permanent discontinuation of the study treatment and/or the AEs that led to premature discontinuation of study treatment.

Any unexpected and serious adverse event for which there is a reasonable possibility that the product caused the event, in accordance with 21 CFR 312.32 will be reported to the FDA within 7 days.

\section{Baseline (before treatment) and follow-up tests}

a. Medical History and Physical Examination including vital signs, height and weight. Performance status. Complete blood count $(\mathrm{CBC})$ with differential and platelet count. Serum Chemistries: transaminases (AST, ALT), alkaline phosphatase, total and direct bilirubin, creatinine, albumin, serum creatinine, acute phase reactants, IL-6, Protein C, D-dimer. To be performed daily and/or as needed during the treatment period.

b. EKG (baseline and as needed).

c. Chest Xray or CT scan (baseline and as needed)

Follow up and evaluation during and post treatment

During vector infusion and follow-up, the patient will be closely monitored for adverse events or changes in clinical status. The patient will be closely followed as an inpatient or outpatient at regular intervals.

\section{Collection of specimens for analysis and archiving}

a. Replication-competent retrovirus (RCR) in PBL's: $5 \mathrm{ml}$ blood (in CPT vacutainer) before, 8 weeks, 6 months, 1 year after vector infusions have been completed, and yearly thereafter. If all post-infusion assays are negative, yearly samples will be archived for 15 years.

b. Vector DNA PCR analysis on PBLs: $5 \mathrm{ml}$ blood (in CPT vacutainers). Analysis will be performed before vector infusion, 8 weeks, 6 months, 1 year after vector infusions are completed. If all post-vector infusion assays are negative, yearly samples will be archived for 15 years.

c. Archived specimens: $5 \mathrm{ml}$ blood(in a red-top tube) will be archived for further testing at each time point indicated above.

\section{Stopping rules}

The NCI Common Toxicity Criteria (CT-CAE version 5.0) will be used to achieve consistency in response to drug/intervention toxicities. Toxicities will be graded on a 1 to 5 grading scale.

If the patient develops a treatment-related $>$ Grade 3 adverse event (CTCAE Vs 5.0), the DeltaRex-G infusions will be held and the patient will be monitored until the toxicity has resolved to $<$ Grade 1 , and the patient is stable, after which treatment may be resumed. If the adverse event does not resolve to $<$ Grade 1 within 3 days, the DeltaRex-G treatment will be held until the data are discussed with the Food and Drug Administration (FDA).

\section{General management of adverse events}

a. All serious therapy-related or unexpected adverse events will be reported to the FDA and the IRB within 7 days of the incident. All other adverse events will be reported to the FDA and IRB in annual report format and in the final study report.

For Grade III adverse events not related to vector infusions, the investigators will discuss the various options available. The 
appropriate action relative to the patient with a Grade III adverse event will be evaluated. If appropriate, a decision of whether the patient shall continue vector infusions will be made. In the event of death, permission to perform an autopsy will be requested.

\section{b. Vector-related toxicities}

The risks associated with retrovector infusion include development of replication competent retrovirus, vector neutralizing antibodies, vector integration in non-target organs. Acute toxicity may occur as outlined in the common toxicity criteria, from infusional reactions or or from unknown vector toxicity.

All Grade III or IV toxicities, whether or not they are attributable to the DeltaRex-G, will be reported in the clinical study summary report. In the event of death, an autopsy report will be submitted if a post-mortem examination was conducted.

\section{Reporting the Results}

Consistent with 21 CFR 312.310 (c) (2), at the conclusion of the treatment (i.e., at the completion of follow-up, a written summary of the results of the treatment including adverse events will be provided to FDA.

\section{Discussion}

COVID-19 disease is classified by the U.S. National Institutes of Health as mild, moderate, severe, and critical. In mild disease, affected patients have reported symptoms such as fever, cough, loss of taste or smell, myalgia, diarrhea, and vomiting without dyspnea or any abnormality on chest imaging. Moderate disease is defined as having shortness of breath or abnormal chest imaging but with saturation levels ( $\mathrm{SpO} 2) \geq 94 \%$. Patients with COVID-19 disease are considered to have severe disease if they have low oxygen saturation $(\mathrm{SpO} 2)<94 \%$, findings of ARDS $((\mathrm{PaO} 2 / \mathrm{FiO} 2)<300 \mathrm{~mm} \mathrm{Hg})$, respiratory rate $>30 / \mathrm{min}$, or diffuse lung infiltrates found on more than half of the total lung volume on chest imaging. If patients have respiratory failure, septic shock, multiple organ dysfunction, or failure, then the condition of patients is considered critical [20]. Though the current classification of COVID-19 is centered around clinical presentation and radiological findings, several prognostic markers are often routinely followed across institutions (procalcitonin, D-Dimer, Fibrinogen, LDH, Ferritin) and may be incorporated in characterizing severity of illness as the current diagnostic approach evolves.

Clinical management of COVID-19 consists of immediate stabilization of oxygen requirements depending on disease severity with the use of nasal cannula, non-invasive ventilation and invasive mechanical ventilation, and in specific cases- ExtraCorporeal Membrane Oxygenation (ECMO). Though pharmacological management of COVID-19 also depends on severity of illness, there has been a shifting paradigm highly dependent on agents that received FDA-approval or Emergency Use Authorization, availability, and general cost. Additionally, there has been an increasing emphasis on preventative strategies such as proning measures, antimicrobial therapy in patients demonstrating high susceptibility to superimposed pneumonia, early diuresis to help slow progression of ARDS, and thrombosis prevention with varying approaches to chemical prophylaxis across institutions.

The only FDA-approved antiviral treatment against SARSCoV2 currently is Remdesivir [21]. As an adenosine analog,
Remdesivir inhibits RNA-dependent RNA polymerase selectively. In clinical trials, Remdesivir has shown reduction of hospitalization stay with approval by FDA to treat hospitalized COVID-19 patients older than 12 years old. [21-23] Baricitinib, an anti-inflammatory and antiviral drug has been granted Emergency Use Authorization by FDA for COVID-19 patients who are hospitalized and require oxygen or respiratory support as well [24]. By inhibiting Janus kinase, Baricitinib prevents the transmission of cytokine stimuli into the cell, preventing excessive cytokine stimulation that leads to severe and critical COVID-19 disease [25].

FDA also granted Emergency Use Authorization for several monoclonal antibody treatments including sotrovimab [26] and the combination of casirivimab and imdevimab [REGN-COV2, 27] to treat mild to moderate COVID-19 disease in patients older than 12 years old and carrying a high risk of progression to severe disease. Although the exact mechanism of action of sotrovimab is not known, it is thought that sotrovimab binds to the spike protein of SARS-CoV-2 and prevents the virus from entering the cell [26]. REGN-COV2 bind to a different site of the spike protein and show efficacy activity by blocking the virus from attaching to the human ACE2 receptor [28]. FDA also authorized casirivimab and imdevimab combination as prophylaxis for people who have been exposed to COVID-19 or have a high risk of exposure and carry a high probability for disease progression [28].

The US National Institutes of Health recommends dexamethasone for hospitalized patients requiring any level of oxygen support due to severe COVID-19 disease [16]. Tocilizumab, an IL-6 receptor blocker, was issued an Emergency Use Authorization by FDA for patients older than 2 years old and who have severe COVID-19 disease requiring oxygen supplementation or respiratory support and receiving corticosteroid therapy [29].

The current use of approved therapeutic agents remains limited by financial cost, availability and agent-specific dose toxicities as determined by a patient's baseline comorbidities, susceptibility to multiorgan dysfunction, and general clinical context of the patient. Currently, the literature suggests the use of dexamethasone and Remdesivir to be the most consistent standardized treatment approach to hospitalized COVID-19 patients across healthcare centers in the United States, with consideration for other therapeutic agents as deemed appropriate by healthcare providers on a case-by-case basis. The addition of Remdisivir to dexamethasone has been encouraged in patients demonstrating increasing severity of COVID-19 [20]. There remains no standardized approach individualized to patients with severe COVID-19 disease however, and the current treatment paradigm will inevitably evolve as our understanding of the pathogenesis and clinical course of COVID-19 becomes more refined.

Deltarex-G is the only gene therapy product authorized for emergency use by the US FDA for a patient with severe COVID-19 (IND\# 27185). In this case, the patient who had initially desaturated requiring 10L oxygen, and did not respond initially to high dose steroids and Remdesivir, eventually improved before DeltaRex-G could be shipped to the treating hospital. Nevertheless, the FDA EUA of DeltaRex-G for a patient with severe COVID-19 establishes a precedent as a promising therapy 
for many other patients who would not survive the onslaught of severe COVID-19/ARDS.

\section{Conclusion}

It has been shown in many publications and studies that the cytokine release syndrome is an important cause of mortality and morbidity in COVID-19 induced ARDS. DeltaRex-G could work in reducing excessive inflammation and cytokine storm by inhibiting the proliferation of immune cells, reducing cytoking release, and thus hastening patient recovery time and improving survival. A Phase I/II clinical trial is pending to determine the safety and efficacy of DeltaRex-G in 36-40 patients with COVID-19 induced ARDS (NCT04378244), following which a Phase III clinical trial will be conducted to obtain marketing approval for DeltaRex-G for severe COVID-19/ARDS.

\section{Abbreviations}

ARDS: Acute Respiratory Distress Syndrome; CFU: Colony-Forming Units; DLE: Damaged Lung Environment; DRG-101:DeltaRex-G vector; EKG: Electrocardiogram; EUA: Emergency Use Auhthorization; FDA: US Food and Drug Administration; MLV: Murine Leukemia Virus; PRN: Per Needed; po: By Mouth; TEAE: Treatment Emergent Adverse Events; TME: Tumor Microenvironment; XC: Exposed Collagen

\section{Conflicts of Interest}

Dr. Erlinda M. Gordon and Dr. Frederick L. Hall are co-inventors of DeltaRex-G, including its targeted gene delivery system, which was originally developed at the University of Southern California Keck School of Medicine, and they are co-founders of Delta Next-Gene, LLC. The other authors have no competing interests.

\section{References}

1. [No Authors Listed]. Centers for Disease Control and Prevention COVID Data Tracker Weekly Review. https://www.cdc.gov/coronavirus/2019-ncov/covid-data/covidview/index.html Accessed [August 31].

2. Elezkurtaj S, Greuel S, Ihlow J, et al. Causes of death and comorbidities in hospitalized patients with COVID-19. Sci Rep. 2021; $11: 4263$.

3. Tzotzos SJ, Fischer B, Fischer H, et al. Incidence of ARDS and outcomes in hospitalized patients with COVID-19: a global literature survey. Crit Care. 2020; 24:516.

4. Fernández-Francos S, Eiro N, González-Galiano N, et al. Mesenchymal Stem Cell-Based Therapy as an Alternative to the Treatment of Acute Respiratory Distress Syndrome: Current Evidence and Future Perspectives. Int J Mol S. 2021; 15:7850.

5. Ragab D, Salah Eldin H, Taeimah M, et al. The COVID-19 Cytokine Storm; What We Know So Far. Front Immunol. 2020;11:1446.

6. Ye Q, Wang B, Mao J. The pathogenesis and treatment of the 'Cytokine Storm' in COVID-19. J Infect. 2020;80(6):607-613.

7. Huang C, Wang Y, Li X, et al. Clinical features of patients infected with 2019 novel coronavirus in Wuhan, China [published correction appears in Lancet. 2020;395(10223):497-506
8. Ward SE, Fogarty H, Karampini E, et al. ADAMTS13 regulation of VWF multimer distribution in severe COVID-19. J Thromb Haemost. 2021;19(8):1914-1921.

9. Caramaschi S, Kapp ME, Miller SE, et al. Histopathological findings and clinicopathologic correlation in COVID-19: a systematic review. Mod Pathol. 2021;34(9):1614-1633.

10. Wood SL, Pernemalm M, Crosbie PA, et al. The role of the tumor-microenvironment in lung cancer-metastasis and its relationship to potential therapeutic targets. Cancer Treat Rev. 2014;40(4):558-566.

11. Chawla SP, Bruckner H, Morse MA, et al. A Phase I-II Study Using Rexin-G Tumor-Targeted Retrovector Encoding a Dominant-Negative Cyclin G1 Inhibitor for Advanced Pancreatic Cancer. Mol Ther Oncolytics. 2018;12:56-67.

12. Gordon EM, Lopez FF, Cornelio GH, et al. Pathotropic nanoparticles for cancer gene therapy Rexin-G IV: three-year clinical experience. Int J Oncol. 2006;29(5):1053-1064.

13. Hall FL, Liu L, Zhu NL, et al. Molecular engineering of matrix-targeted retroviral vectors incorporating a surveillance function inherent in von Willebrand factor. Hum Gene Ther. 2000;11(7):983-993.

14. Chawla SP, Chawla NS, Quon D, et al. An Advanced Phase 1/2 Study using an XC-Targeted Gene Therapy Vector for Chemotherapy Resistant Sarcoma. Sarcoma Res Int. 2016; 3(1): 1024.

15. Gordon EM, Hall FL. Noteworthy clinical case studies in cancer gene therapy: tumor-targeted Rexin-G advances as an efficacious anti-cancer agent. Int J Oncol. 2010;36(6):1341-1353.

16. Chawla SP, Chua VS, Fernandez L, et al. Advanced phase I/ II studies of targeted gene delivery in vivo: intravenous Rexin-G for gemcitabine-resistant metastatic pancreatic cancer. Mol Ther. 2010;18(2):435-441

17. Chawla SP, Chua VS, Fernandez L, et al. Phase I/II and phase II studies of targeted gene delivery in vivo: intravenous Rexin-G for chemotherapy-resistant sarcoma and osteosarcoma. Mol Ther. 2009;17(9):1651-1657.

18. [No Authors Listed]. Study to Evaluate the Safety and Antiviral Activity of Remdesivir (GS-5734TM) in Participants with Severe Coronavirus Disease (COVID-19) - Full-Text View." ClinicalTrials.gov. https://clinicaltrials.gov/ct2/show/NCT04292899 Accessed [August 31].

19. Gordon EM, Cornelio GH, Lorenzo CC 3rd, et al. First clinical experience using a 'pathotropic' injectable retroviral vector (Rexin-G) as intervention for stage IV pancreatic cancer. Int $\mathbf{J}$ Oncol. 2004;24(1):177-185.

20. [No Authors Listed]. Coronavirus Disease 2019 (COVID-19) Treatment Guidelines. https://www.covid19treatmentguidelines.nih.gov/ Accessed [August 31]

21. [No Authors Listed]. FDA Approves First Treatment FOR COVID-19. https://www.fda.gov/news-events/press-announcements/fda-approves-first-treatment-covid-19 Accessed [August 31]

22. Singh AK, Singh A, Singh R, et al. Remdesivir in COVID-19: A critical review of pharmacology, pre-clinical and clinical studies. Diabetes Metab Syndr. 2020;14(4):641-648 
23. Beigel JH, Tomashek KM, Dodd LE, et al. Remdesivir for the Treatment of Covid-19 - Final Report. N Engl J Med. 2020;383(19):18131826.

24. An EUA for baricitinib (Olumiant) for COVID-19. Med Lett Drugs Ther. 2020;62(1614):202-203

25. Jorgensen SCJ, Tse CLY, Burry L, et al. Baricitinib: A Review of Pharmacology, Safety, and Emerging Clinical Experience in COVID-19. Pharmacotherapy. 2020;40:843-856.

26. [No Authors Listed]. An EUA for sotrovimab for treatment of COVID-19." The Medical letter on drugs and therapeutics vol. 63,1627 (2021): 97-98.
27. Weinrich DM, Sivapalasingam S, Norton T, et al. REGN-COV, a Neutralizing Antibody Cocktail, in Outpatients with Covid-19. N Eng J Med. 2021;384: 238-251. DOI:10.1056/NEJMoa2035002

28. [No Authors Listed]. An EUA for casirivimab and imdevimab for COVID-19." The Medical letter on drugs and therapeutics vol. 62,1614 (2020): 201-202.

29. [No Authors Listed]. The Medical letter, inc. An EUA For Tocilizumab (ACTEMRA) for COVID-19. https://secure.medicalletter. org/w1629a Accessed [August 31]

To cite this article: Soheili E, Michel G, Yerli BA, et al. Larkin: FDA Emergency Use Authorized Treatment Protocol of DeltaRex-G for Severe Covid-19 [IND\# 27185]. European Journal of Respiratory Medicine. 2021; 3:2.

(C) 2021 Soheili E, et al. 